\title{
Aortic root dimensions as a correlate for aortic regurgitation's severity
}

\author{
Jan-Per Wenzel ${ }^{1,4}\left(\right.$ E Elina Petersen ${ }^{4}$. Julius Nikorowitsch ${ }^{1}$. Jessica Müller ${ }^{4}$. Tilo Kölbel ${ }^{5}$. \\ Hermann Reichenspurner ${ }^{2,3} \cdot$ Stefan Blankenberg ${ }^{1,2,4} \cdot$ Evaldas Girdauskas $^{2,3}$
}

Received: 23 March 2021 / Accepted: 1 July 2021 / Published online: 7 July 2021

(c) The Author(s) 2021

\begin{abstract}
To evaluate the prevalence of aortic regurgitation (AR) and associations between the individual aortic root components and AR severity in the general population. The study included the first 10,000 participants of the population-based Hamburg City Health Study (HCHS) of whom 8259 subjects, aged $62.23 \pm 8.46$ years (51.3\% females), enrolled 2016-2018, provided echocardiographic data. 69 subjects with bicuspid valves and 23 subjects with moderate/severe aortic stenosis were excluded. Aortic root dimensions were measured using state-of-the-art cardiac ultrasound, including the aortic annulus, sinus of Valsalva, sinotubular junction (STJ), and ascending aorta, in diastole and systole. The distribution of AR was: 932 (11.4\%) mild, $208(2.5 \%)$ moderate, and $20(0.24 \%)$ severe. Patients with moderate or severe AR were predominantly male at advanced age who had hypertension, coronary artery disease, atrial fibrillation, and renal dysfunction. Increasing AR severity correlated with higher absolute and indexed aortic root diameters (e.g., end-diastolic sinus of Valsalva for no-mild-moderate-severe AR in $\mathrm{mm} \pm$ standard deviation: $34.06 \pm 3.81 ; 35.65 \pm 4.13 ; 36.13 \pm 4.74 ; 39.67 \pm 4.61 ; \mathrm{p}<0.001)$. In binary logistic regression analysis, all aortic root components showed significant associations with moderate/severe AR. Mid-systolic STJ showed the strongest association with moderate/severe AR (OR 1.33, 95\% confidence interval 1.25-1.43, p <0.001). AR was prevalent in $14.2 \%$, of whom $2.8 \%$ showed moderate/severe AR. All assessed aortic root diameters correlated with the prevalence and severity of AR. STJ diameter had the strongest association with moderate/severe AR possibly reflecting the pathophysiological impact of an increasingly dilated STJ in the context of an ageing aorta.
\end{abstract}

Keywords Aortic root $\cdot$ Echocardiography $\cdot$ Sinotubular junction $\cdot$ Aortic annulus $\cdot$ Aortic regurgitation $\cdot$ Hamburg City Health Study

\section{Abbreviations}

ACEi

Asc

Ao

Angiotensin-converting enzyme inhibitor

Ascending

Aorta/aortic
An

AR

ARB

ASE
Annulus

Aortic regurgitation

Angiotensin receptor blocker

American Society of Echocardiography
Jan-Per Wenzel

ja.wenzel@uke.de

Elina Petersen

e.petersen@uke.de

Julius Nikorowitsch

j.nikorowitsch@uke.de

Jessica Müller

mueller-jessica@gmx.net

Tilo Kölbel

t.koelbel@uke.de

Hermann Reichenspurner

reichenspurner@uke.de

Stefan Blankenberg

s.blankenberg@uke.de
Evaldas Girdauskas

e.girdauskas@uke.de

1 Department of General and Interventional Cardiology, University Heart and Vascular Center Hamburg, Hamburg, Germany

2 German Center for Cardiovascular Research (DZHK), Partner Site Hamburg/Kiel/Luebeck, Hamburg, Germany

3 Department of Cardiovascular Surgery, University Heart and Vascular Center Hamburg, Hamburg, Germany

4 Epidemiological Study Center, Hamburg, Germany

5 Department of Vascular Medicine, German Aortic Center Hamburg University Heart and Vascular Center, Hamburg, Germany 


$\begin{array}{ll}\text { AV } & \text { Aortic valve } \\ \text { BMI } & \text { Bodyss mass index } \\ \text { BSA } & \text { Body surface area } \\ \text { CRP } & \text { C-reactive protein } \\ \text { EACVI } & \text { European Association of Cardiovascular } \\ & \text { Imaging } \\ \text { ED } & \text { End-diastolic/diastole } \\ \text { ESC } & \text { European Society of Cardiology } \\ \text { GFR } & \text { Glomerular filtration rate } \\ \text { HCHS } & \text { Hamburg City Health Study } \\ \text { IMT } & \text { Intima media thickness } \\ \text { IVST } & \text { Interventricular septum thickness } \\ \text { LAVI } & \text { Left atrial systolic volume indexed to body } \\ & \text { surface area } \\ \text { LVEDD } & \text { Left ventricular end-diastolic diameter } \\ \text { LVEDV } & \text { Left ventricular end-diastolic volume } \\ \text { LVEF } & \text { Left ventricular ejection fraction } \\ \text { MS } & \text { Mid-systolic/systole } \\ \text { NT-proBNP } & \text { N-terminal pro-B-type natriuretic peptide } \\ \text { NYHA } & \text { New York Heart Association } \\ \text { SoV } & \text { Sinus of Valsalva } \\ \text { STJ } & \text { Sinotubular junction } \\ \text { TAPSE } & \text { Tricuspid annular peak systolic excursion }\end{array}$

\section{Introduction}

The aortic root is a complex and dynamic entity, varying in size proportionally to height, weight, and age. The interplay of its four different parts, the aortic annulus (AoAn), sinus of Valsalva (SoV), sinotubular junction (STJ), and the proximal ascending aorta (AscAo), is crucial for aortic valve (AV) competence. Along with a dramatic decrease of rheumatic heart disease in the Western world, aortic root dilatation evolved into the predominant cause of aortic regurgitation (AR) [1-4]. However, AR is a multifactorial valvular disease and the correlation between the extent of aortic root dilatation and the severity of AR remains controversial [2, 3]. Notably, most previously published studies correlating AR with aortic root enlargement focused on the aortic root as one entity. Accordingly, little is known about the role of the individual components of the aortic root and their timepoint of measurement during the heart cycle in relation to AR, which is crucial to understand in the field of AV repair surgery. Furthermore, given major improvements in spatial and temporal resolution of 2-dimensional and color Doppler transthoracic echocardiography (TTE), most previous studies investigating AR prevalence and its correlation with aortic root diameter are rather outdated [2, 5-7].

Hence, first we aimed at providing up-to-date data on the prevalence of AR as assessed by state-of-the-art TTE in the general population. Second, we investigated the correlation between AR severity and each individual part of the aortic root measured systematically in end-diastole and mid-systole.

\section{Methods}

\section{Study setting}

The study population derived from a sample of 10,000 consecutive, at random selected participants from the Hamburg City Health Study (HCHS, www.hchs.hamburg) who underwent TTE. As previously described, the HCHS is a singlecenter, prospective, long-term, population-based cohort study [8]. HCHS aims to evaluate the interaction of socioeconomic risk factors, modern imaging techniques, physiological measurements, and clinical variables. All measurements were conducted between 2016 and 2018 during a one-day baseline visit at the HCHS Epidemiological Study Center Hamburg-Eppendorf, Germany according to the published study protocol [8]. Demographics and clinical parameters were assessed by standardized interviews conducted by specifically trained medical professionals following standard operating procedures as well as self-reported questionnaires [8]. Blood samples were withdrawn under fasting conditions and all subjects underwent biomarker quantification including NT-proBNP. After application of the exclusion criteria (1) incompletely recorded images or insufficient image quality of TTE for standardized measurements (1741) (2) moderate/severe aortic stenosis (23) or (3) bicuspid valve disease (69), 8167 subjects were included in the study. The study protocol was approved by the local ethics committee (PV5131, Medical Association Hamburg) and the HCHS steering board. All participants gave written informed consent. The investigation conforms with the principles outlined in the Declaration of Helsinki.

\section{TTE image acquisition and analysis}

TTE examinations were performed and analyzed by professional cardiologists and sonographers (technicians) at the baseline visit on dedicated ultrasound machines (Siemens Acuson SC2000 Prime, Siemens Healthineers, Erlangen, Germany) following standard operating procedures at the HCHS Epidemiological Study Center Hamburg-Eppendorf, Hamburg, Germany [8]. All TTE standard views were assessed in 2-dimensional echocardiography, including a 3-dimensional four-chamber view for chamber quantification. For continuous quality assessment, every 100th TTE exam was analyzed twice by an ESC TTE certified cardiologist. Qualitative and quantitative image analyses were performed using an off-line workstation with the commercially available and established Siemens syngo SC2000 software (Siemens syngo SC 2000 Version 4.0, Siemens 
Healthineers, Erlangen, Germany) in agreement with the current recommendations of the American Society of Echocardiography (ASE) and the European Association of Cardiovascular Imaging (EACVI) [9, 10]. Left sided volumes and ejection fraction (LVEF) were calculated using from two-dimensional and three-dimensional loops using the method of disks summation. Left-sided diameters were measured in parasternal long-axis view. Mitral inflow pattern was assessed in apical four-chamber view by placing pulsed-wave (PW) Doppler sample volume between mitral leaflet tips. PW tissue Doppler imaging (TDI) e' velocity was measured in apical four-chamber view by placing the sample volume at the lateral and septal basal regions. Tricuspid annular plane systolic excursion (TAPSE) was obtained by M-mode echocardiography in the apical four-chamber view. Right ventricular fractional area change was assessed in a right ventricular focused four-chamber view. Left atrial global peak systolic strain was measured in apical fourchamber view by velocity vector imaging averaging global peak strain of all segments of the left atrium.

\section{Aortic root and valve assessment}

For aortic root assessment, a zoomed transthoracic parasternal long axis view was recorded at breath hold [10]. Systematic measurements of the aortic root were performed perpendicular to the proximal aorta axis in end-diastole (ED) as well as in mid-systole (MS) including the following: The (a) AoAn, (b) SoV, (c) STJ, and (d) AscAo (at $2 \mathrm{~cm}$ range from the STJ) were measured perpendicular to the proximal aorta axis. AoAn, STJ and AscAo were measured using the inneredge to inner-edge convention while the SoV was measured using the leading-edge to leading-edge convention.

AV morphology (i.e., bicuspid vs. tricuspid) and potential degenerative changes were assessed in the parasternal long axis, AV short axis and apical views. Aortic stenosis and regurgitation were assessed according to current ESC guidelines [11]. An in-depth description of valve assessment is provided in the supplements.

\section{Statistical analysis}

Continuous variables are given as mean \pm standard deviation (SD). Categorical variables are given as absolute numbers and percentage of participants. Multiple group comparisons of different severities of AR were assessed by analysis of variance (ANOVA) for continuous variables and chi-squared test $\left(\chi^{2}\right)$ for categorical variables. BSA was calculated using the DuBois formula. Aortic root measurements were divided by BSA to obtain indexed measurements. Correlation of these indexed measurements with AR severity as well as the ratio of sinotubular junction and aortic annulus were presented as boxplots using ANOVA to draw multiple group comparisons.

AR was dichotomized into "no AR" and "moderate/ severe AR" for logistic regression analyses. We constructed a model including all aortic root measurements. However, substantial multicollinearity between measures of aortic root was detected using the variance inflation factor (VIF). Therefore, we constructed separate models for all eight aortic root measurements. Age, sex, BSA, hypertension, and diabetes were pre-defined as relevant confounders and controlled for in all aortic root models. Results of logistic regression were corrected post-hoc using the Holm method and are depicted by odds ratios (OR) and corresponding $95 \%$ confidence intervals (CI). Differences were considered statistically significant at a two-sided p-value level of 0.05 after post-hoc correction. All statistical analyses were performed using $\mathrm{R}$ (version 3.6.2). A list of the used packages and versions can be found in the appendix.

\section{Results}

The analyzed study population included 8167 HCHS subjects of the first $10,000 \mathrm{HCHS}$ participants, with 4191 female $(51.3 \%)$ and a mean age of $62.23 \pm 8.46$ years (range 45-74 years) (Table 1; Fig. 1). 23 subjects were excluded due to moderate/severe AS and 69 due to bicuspid aortic valve morphology. While 7007 (85.8\%) subjects were free of AR, 932 (11.4\%) showed mild, 208 (2.5\%) moderate and $20(0.3 \%)$ severe AR. Patients with moderate or severe AR showed predominantly male sex, a higher age, and had more hypertension, CAD, atrial fibrillation, hypercholesterolemia, NT-proBNP elevation, and renal dysfunction compared with patients with no AR. Weight, height, heart rate, BSA, Glucose, triglycerides, hsCRP, hemoglobin, and diabetes did not show significant intergroup differences. The use of ACE inhibitors, angiotensin receptor blockers, beta-blockers, diuretics, and statins was more common in subjects with moderate/severe AR. Echocardiography revealed slightly lower left ventricular systolic function and larger left-sided cavities as well as a higher E/e' ratio in subjects with moderate/severe AR compared to subjects without AR (Table 2). 7 subjects suffered from moderate/severe AR combined with mild AS. The prevalence of moderate/severe MR increased with AR severity.

Inter- and intraobserver reproducibility of echocardiographic aortic root assessment, derived from the measurements of a random sample of 100 exams measured by three different observers, were remarkably high for all variables, as shown by intraclass correlation coefficients ranging from 0.92 to 0.99 (Supplemental Table 1). Systolic were greater than diastolic measurements of the aortic root. Both the absolute and indexed diameters of 
Table 1 Baseline Characteristics of the study population stratified by AR severity

\begin{tabular}{|c|c|c|c|c|}
\hline & No AR $(n=7007)$ & Mild AR (n=932) & Moderate/Severe AR $(n=228)$ & p-value \\
\hline \multicolumn{5}{|l|}{ Demographics and biological data } \\
\hline Age, years & $61.6 \pm 8.4$ & $66.0+7.8$ & $67.3 \pm 7.4$ & $<0.001$ \\
\hline Male & $3369(48.1)$ & $482(51.7)$ & $125(54.8)$ & 0.019 \\
\hline Weight, kg & $78.6 \pm 16.2$ & $78.1 \pm 15.3$ & $77.5 \pm 16.5$ & 0.423 \\
\hline Height, $\mathrm{cm}$ & $171.3 \pm 9.5$ & $171.2 \pm 9.3$ & $170.3 \pm 9.9$ & 0.359 \\
\hline $\mathrm{BSA}, \mathrm{m}^{2}$ & $1.9 \pm 0.2$ & $1.9 \pm 0.2$ & $1.9 \pm 0.2$ & 0.34 \\
\hline Systolic blood pressure, $\mathrm{mmHg}$ & $138.4 \pm 19.2$ & $140.9 \pm 19.6$ & $143.5 \pm 18.4$ & $<0.001$ \\
\hline Heart rate, bpm & $69.8 \pm 11.2$ & $69.1 \pm 10.5$ & $69.6 \pm 11.9$ & 0.208 \\
\hline Current smoking & $1438(20.6)$ & $114(12.3)$ & $32(14.1)$ & $<0.001$ \\
\hline NYHA, II/III & $542(8.6)$ & $84(10.0)$ & $20(10.5)$ & 0.267 \\
\hline \multicolumn{5}{|l|}{ Comorbidities } \\
\hline Hypertension & $4292(64.7)$ & $638(70.3)$ & $171(78.1)$ & $<0.001$ \\
\hline Diabetes & $546(8.5)$ & $68(7.7)$ & $17(7.9)$ & 0.728 \\
\hline Coronary artery disease & $291(5.9)$ & $45(6.9)$ & $23(14.9)$ & $<0.001$ \\
\hline Atrial fibrillation & $341(5.4)$ & $75(8.9)$ & $28(13.4)$ & $<0.001$ \\
\hline Peripheral artery disease & $207(3.2)$ & $41(4.9)$ & $8(4.1)$ & 0.042 \\
\hline \multicolumn{5}{|l|}{ Medication } \\
\hline Beta-blockers & $1114(16.7)$ & $180(20.0)$ & $51(23.3)$ & 0.003 \\
\hline Diuretics & $152(2.3)$ & $28(3.1)$ & $8(3.7)$ & 0.15 \\
\hline Statines & $1132(17.0)$ & $182(20.3)$ & $53(24.2)$ & 0.002 \\
\hline ACE/AT-I-antagonists & $1356(20.4)$ & $207(23.1)$ & $63(28.8)$ & 0.003 \\
\hline \multicolumn{5}{|l|}{ Laboratories } \\
\hline $\mathrm{LDL}, \mathrm{mg} / \mathrm{dl}$ & $121.0[96.0,146.0]$ & $118.0[93.0,143.0]$ & $119.0[96.0,150.0]$ & 0.058 \\
\hline $\mathrm{GFR}, \mathrm{ml} / \mathrm{min}$ & $86.3[75.5,94.7]$ & $83.1[71.9,90.7]$ & $81.7[71.4,88.2]$ & $<0.001$ \\
\hline NT-proBNP, ng/l & $77.0[43.0,141.0]$ & $100.0[54.3,179.5]$ & $136.0[79.0,228.0]$ & $<0.001$ \\
\hline hsCRP,mg/l & $0.12[0.06,0.26]$ & $0.12[0.06,0.24]$ & $0.12[0.06,0.26]$ & 0.428 \\
\hline Glucose, mg/dl & $92.0[86.0,100.0]$ & $92.0[96.0,99.0]$ & $93.0[87.0,100.0]$ & 0.562 \\
\hline
\end{tabular}

Data are given as $\mathrm{n}(\%)$ or mean \pm standard deviation, $\mathrm{p}$-value for intergroup differences

$A C E i$ angiotensin-converting enzyme inhibitor, $A R$ aortic regurgitation, $A R B$ angiotensin receptor blocker, $B M I$ body mass index, $B S A$ body surface area, $C A D$ coronary artery disease; $h s C R P$ high sensitivity C-reactive protein, GFR glomerular filtration rate, NT-proBNP N-terminal proB-type natriuretic peptide, NYHA New York Heart Association

aortic root components showed the lowest values in the no AR group and the highest values in the severe AR group (Table 3; Fig. 2). In binary logistic regression, age was significantly associated with AR prevalence (OR 1.08, 95\% CI 1.06-1.10, p <0.001). Furthermore, both in univariate as well as in multivariate logistic regression analysis, adjusted for age, sex, BSA, hypertension, and diabetes, significant associations were detected for all end-diastolic and mid-systolic aortic root variables with mild as well as with moderate/severe AR, even after correcting for multiple testing (Table 4, Supplementary Table 2, Fig. 3, Supplementary Fig. 1 and 2). The exclusion of subjects with mild AS did not change the reported associations with aortic root diameters (Supplementary Table 3). The strongest association for the correlation with moderate/severe AR was found for MS STJ (OR 1.33, 95\% confidence interval $1.25-1.43, \mathrm{p}<0.001)$.

\section{Discussion}

The present study analyzes standardized, state-of-the-art echocardiographic aortic root measurements in association with the severity of aortic regurgitation in a large sample of the population-based HCHS. Major findings include that AR is common in the general population and that the size of all parts of the aortic root are associated with moderate/severe AR. Of the different aortic root parts, MS STJ size showed the strongest correlation with moderate/severe AR.

\section{Prevalence of aortic regurgitation (AR)}

The overall prevalence of AR in the population-based HCHS cohort, aged 45 to 74 years, was $14.2 \%$, while the rate of moderate/severe AR was $2.8 \%$. Subjects with moderate/severe AS or bicuspid valves were excluded from 


\section{STUDY FLOW CHART Aortic root dimensions as a correlate for aortic regurgitation's severity}

\section{HCHS Study Population \\ 45,000 inhabitants of the city of Hamburg between 45 and 74 years}

\section{Study Cohort Sample of the first 10,000 participants}

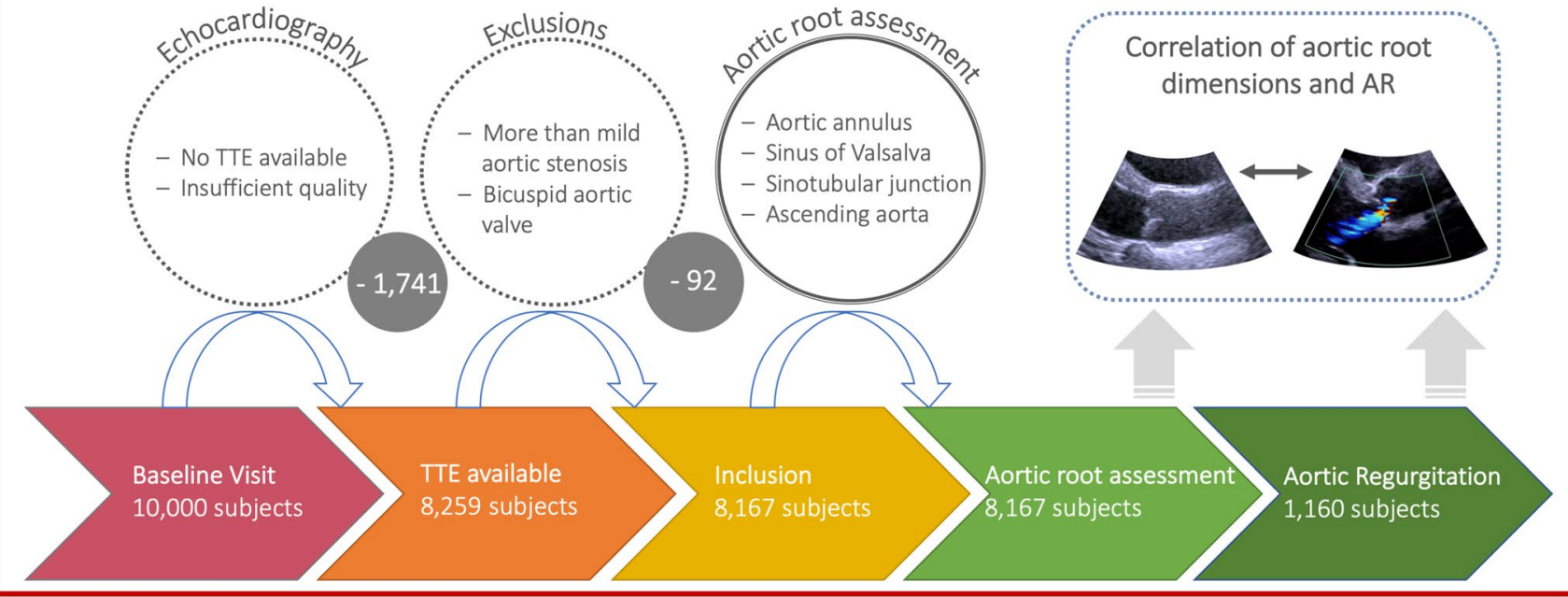

Fig. 1 Study flow-chart. From a total of 8259 subjects providing echocardiographic data, 92 were excluded due more than mild aortic stenosis or bicuspid aortic valve. Consequently, 8167 subjects were included in the study analysis. Of those, 1160 subjects suffered from aortic regurgitation. The aortic root was systematically measured in end-diastole and mid-systole and the correlation between aortic root dimensions and the severity of aortic regurgitation was calculated. $A R$ aortic regurgitation, HCHS Hamburg City Health Study, TTE transthoracic echocardiography
Table 2 Echocardiographic findings of the study population stratified by AR severity

\begin{tabular}{llllr}
\hline & No AR $(\mathrm{n}=7007)$ & Mild AR $(\mathrm{n}=932)$ & $\begin{array}{l}\text { Moderate/Severe } \\
\text { AR }(\mathrm{n}=228)\end{array}$ & p-value \\
\hline Concomitant valvular disease & & & \\
Mild AS & $86(1.3)$ & $23(2.8)$ & $7(3.4)$ & $<0.001$ \\
Moderate/severe MR & $189(2.3)$ & $74(7.9)$ & $19(8.3)$ & $<0.001$ \\
Moderate/severe TR & $623(8.9)$ & $193(20.7)$ & $46(20.2)$ & $<0.001$ \\
Echocardiographic data & & & & \\
LVEF 2D, \% & $58.5 \pm 5.2$ & $58.3+5.0$ & $57.6 \pm 5.6$ & 0.045 \\
LVEF 3D, \% & $59.6 \pm 6.8$ & $59.2 \pm 6.6$ & $58.3 \pm 6.8$ & 0.178 \\
LVEDD, mm & $47.7 \pm 5.2$ & $47.9 \pm 5.3$ & $48.9 \pm 6.4$ & 0.004 \\
LVEDV, ml & $114.9 \pm 31.8$ & $108.4 \pm 30.6$ & $118.9 \pm 34.6$ & $<0.001$ \\
TAPSE, mm & $24.4 \pm 4.3$ & $23.8 \pm 4.4$ & $23.4 \pm 4.2$ & 0.001 \\
RV FAC, \% & $43.2 \pm 8.0$ & $43.7 \pm 7.7$ & $42.7 \pm 8.4$ & 0.217 \\
IVSD, mm & $9.9 \pm 1.7$ & $10.3 \pm 1.8$ & $10.7 \pm 1.9$ & $<0.001$ \\
E/e ' & $7.6 \pm 2.1$ & $7.8 \pm 2.4$ & $8.5 \pm 2.9$ & $<0.001$ \\
LAVI, ml/m ${ }^{2}$ & $27.5 \pm 8.4$ & $27.9 \pm 8.9$ & $30.2 \pm 10.8$ & $<0.001$ \\
LA Global Peak strain, \% & $39.8 \pm 14.4$ & $38.5 \pm 15.4$ & $37.0 \pm 14.5$ & 0.014 \\
\hline
\end{tabular}

Data are given as $\mathrm{n}(\%)$ or mean \pm standard deviation, $\mathrm{p}$-value for intergroup differences. All subjects with moderate/severe aortic stenosis or bicuspid valves were excluded from the analysis

$A S$ aortic stenosis, IVST interventricular septum thickness, $L A$ left atrial, $L A V I$ left atrial volume index, $L V E D D$ left ventricular end-diastolic diameter, $L V E D V$ left ventricular end-diastolic volume, $L V E F$ left ventricular ejection fraction, $M R$ mitral regurgitation, $M S$ mitral stenosis, $R V F A C$ right ventricular fractional area change, TAPSE tricuspid annular peak systolic excursion, $T R$ tricuspid regurgitation 
Table 3 Aortic root diameters absolute $(\mathrm{mm})$ and indexed to body surface area $\left(\mathrm{mm} / \mathrm{m}^{2}\right)$ in relation to AR severity

\begin{tabular}{|c|c|c|c|c|c|}
\hline & None $(n=7007)$ & Mild AR (n=932) & Moderate AR $(\mathrm{n}=208)$ & Severe AR $(n=20)$ & p-value \\
\hline \multicolumn{6}{|l|}{ Absolute } \\
\hline ED Ao annulus & $20.33 \pm 1.71$ & $20.65 \pm 1.84$ & $20.50+2.01$ & $22.26 \pm 1.91$ & $<0.001$ \\
\hline MS Ao annulus & $20.96 \pm 1.76$ & $21.35 \pm 1.87$ & $21.29 \pm 1.98$ & $22.19 \pm 1.85$ & $<0.001$ \\
\hline ED Ao sinus & $34.06 \pm 3.81$ & $35.65 \pm 4.13$ & $36.13 \pm 4.74$ & $39.67 \pm 4.61$ & $<0.001$ \\
\hline MS Ao sinus & $35.02 \pm 3.82$ & $36.15 \pm 4.16$ & $37.16 \pm 4.69$ & $40.88 \pm 3.68$ & $<0.001$ \\
\hline ED Ao STJ & $26.53 \pm 3.07$ & $27.70 \pm 3.25$ & $28.33 \pm 3.63$ & $31.22 \pm 3.27$ & $<0.001$ \\
\hline MS Ao STJ & $27.63 \pm 3.24$ & $29.01 \pm 3.62$ & $30.07 \pm 3.80$ & $33.65 \pm 3.81$ & $<0.001$ \\
\hline ED Asc Ao & $29.94 \pm 3.77$ & $30.96 \pm 4.11$ & $32.56 \pm 4.65$ & $36.03 \pm 3.43$ & $<0.001$ \\
\hline MS Asc Ao & $29.94 \pm 3.51$ & $30.99 \pm 3.81$ & $32.57 \pm 4.12$ & $35.67 \pm 3.37$ & $<0.001$ \\
\hline \multicolumn{6}{|l|}{ Indexed to BSA } \\
\hline ED Ao annulus & $10.79 \pm 1.09$ & $10.97 \pm 1.08$ & $11.03 \pm 1.15$ & $12.18 \pm 1.25$ & $<0.001$ \\
\hline MS Ao annulus & $11.09 \pm 1.08$ & $11.32 \pm 1.05$ & $11.35 \pm 1.20$ & $12.00 \pm 1.15$ & $<0.001$ \\
\hline ED Ao sinus & $18.01 \pm 1.99$ & $18.86 \pm 2.06$ & $19.18 \pm 2.38$ & $21.39 \pm 1.75$ & $<0.001$ \\
\hline MS Ao sinus & $18.54 \pm 2.04$ & $19.23 \pm 2.10$ & $19.69 \pm 2.38$ & $22.30 \pm 1.63$ & $<0.001$ \\
\hline ED Ao STJ & $14.03 \pm 1.66$ & $14.68 \pm 1.77$ & $14.99 \pm 2.00$ & $16.75 \pm 1.55$ & $<0.001$ \\
\hline MS Ao STJ & $14.65 \pm 1.73$ & $15.42 \pm 1.84$ & $15.98 \pm 2.10$ & $18.28 \pm 1.62$ & $<0.001$ \\
\hline ED Asc Ao & $15.79 \pm 2.08$ & $16.40 \pm 2.17$ & $17.10 \pm 2.42$ & $19.56 \pm 2.40$ & $<0.001$ \\
\hline MS Asc Ao & $15.82 \pm 1.96$ & $16.48 \pm 1.89$ & $17.26 \pm 2.04$ & $19.41 \pm 1.29$ & $<0.001$ \\
\hline
\end{tabular}

Data are given as mean \pm standard deviation, $\mathrm{p}$-value for intergroup differences

$A R$ aortic regurgitation, $A s c$ ascending, Ao Aortic/Aorta, BSA Body surface area, ED end-diastolic, $M S$ mid-systolic, $S T J$ sinotubular junction
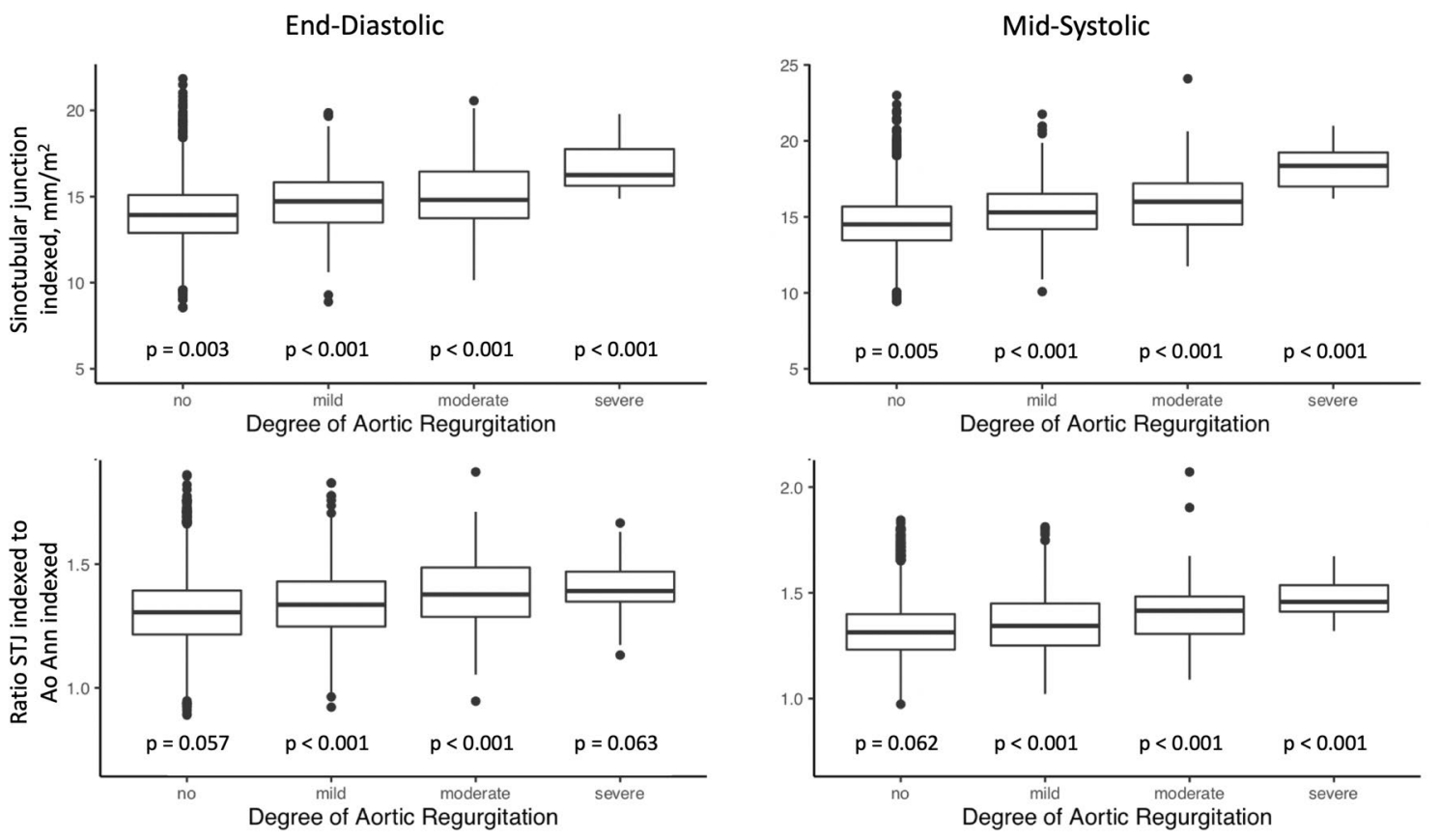

Fig. 2 Correlation of AR severity with the sinotubular junction indexed to BSA as well as the ratio of sinotubular junction indexed and aortic annulus indexed to BSA. $p$-values for inter-group differences. Ao Ann Aortic annulus, BSA body surface area, STJ sinotubular junction

our analysis. Previously published studies on AR prevalence at the populational level reported very heterogeneous results [12-17]. AR prevalence ranged between 1 and 19\%, depending on the baseline characteristics of the analyzed study cohort as well as on the methodology of echocardiographic evaluation. Most previously published studies 
Table 4 Odds ratios and 95\%-CIs derived from univariate and multivariate regression analysis for the associations of aortic root measurements with moderate/severe AR

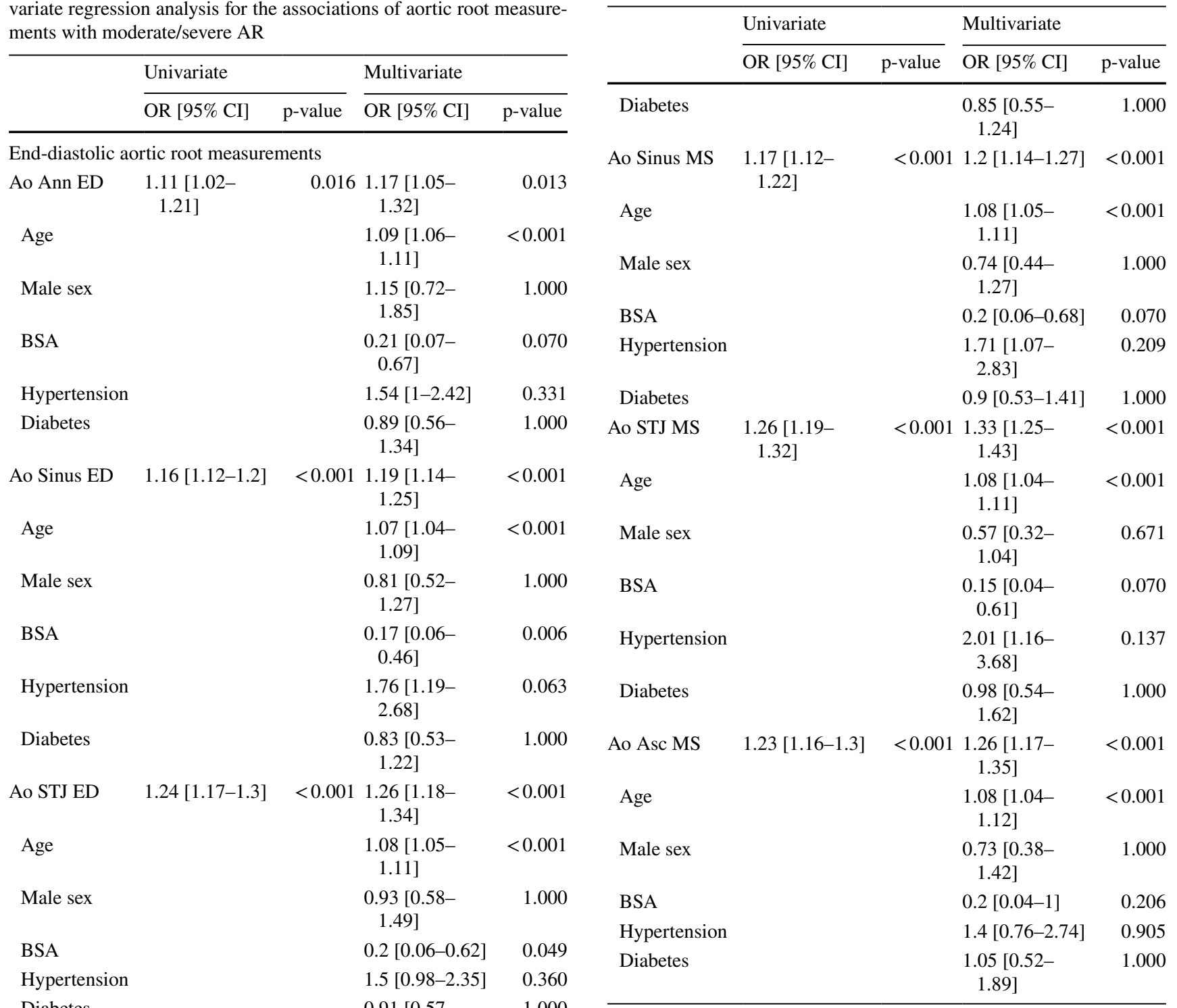

Table 4 (continued)

Mid-systolic aortic root measurements

\begin{tabular}{|c|c|c|c|}
\hline Ao Ann MS & $\begin{array}{c}1.16[1.07- \\
1.26]\end{array}$ & $<0.001 \quad 1.25[1.11-1.4]$ & 0.002 \\
\hline Age & & $1.08[1.05-1.1]$ & $<0.001$ \\
\hline Male sex & & $\begin{array}{c}1.01[0.65- \\
1.55]\end{array}$ & 1.000 \\
\hline BSA & & $\begin{array}{c}0.26[0.09- \\
0.74]\end{array}$ & 0.070 \\
\hline Hypertension & & $\begin{array}{c}1.66[1.12- \\
2.54]\end{array}$ & 0.132 \\
\hline
\end{tabular}

P-values were corrected for multiple testing using the post-hoc Holm method

Ao aortic, $A R$ aortic regurgitation, Asc ascending, $B S A$ body surface area; $C I$ confidence interval, $E D$ end-diastolic, $M S$ mid-systolic, $O R$ odds ratio, $S T J$ sinotubular junction

demonstrated a significant association between age and AR prevalence $[3,13,15]$. While the overall prevalence of $A R$ in younger populations is considerably low, it rises with ageing $[3,12,13]$. In line with this finding, we showed a significant association between age and AR prevalence. Furthermore, in several studies, including the Framingham Cohort Study, AR prevalence was associated with male sex $[15,18]$. Nevertheless, other population-based studies did not find sex-associated differences in AR occurrence $[3,16,17]$. Our study showed in univariate analysis a significantly higher number of male subjects in the AR 


\section{Associations between aortic root dimensions and moderate/severe aortic regurgitation}
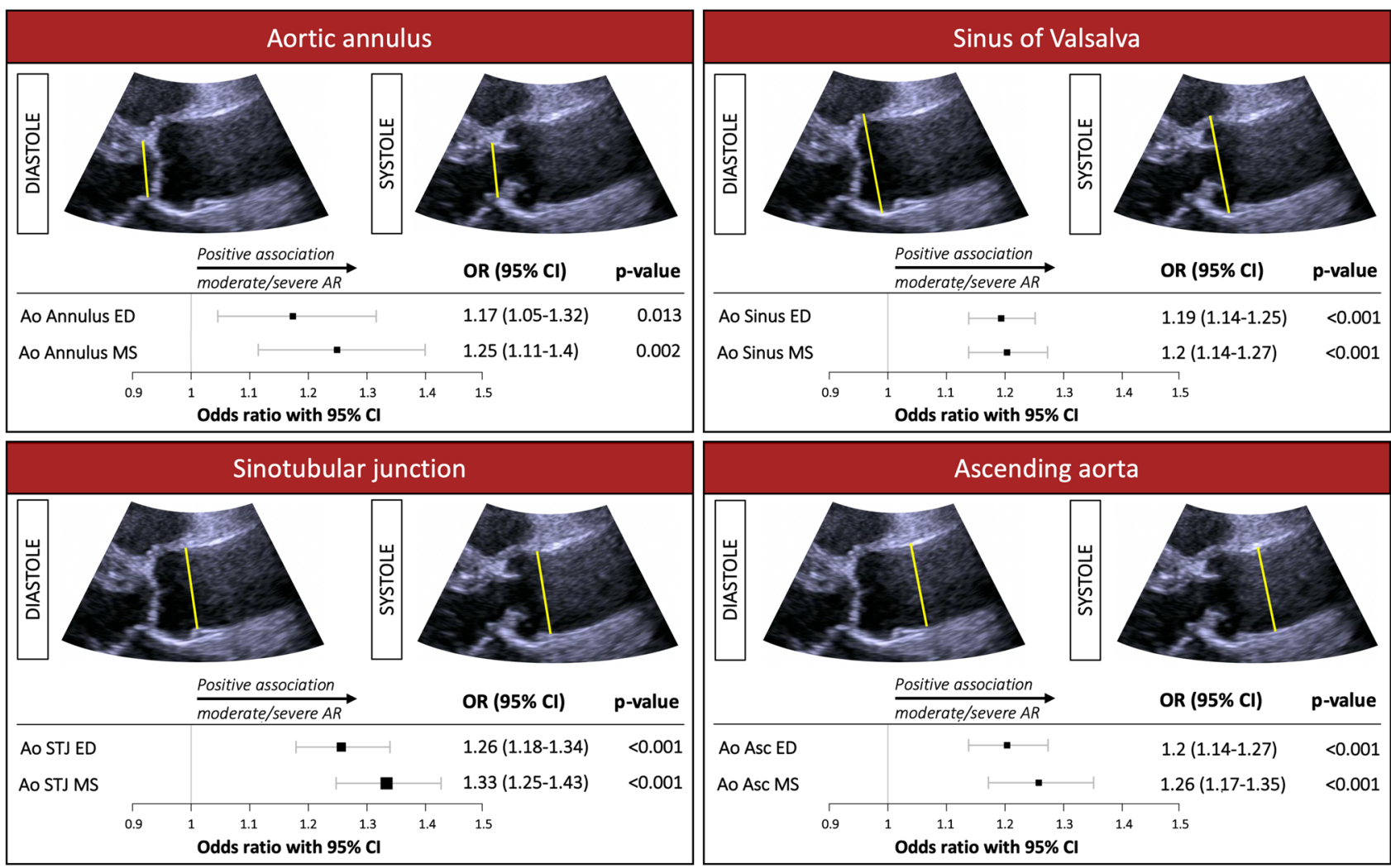

Fig. 3 Associations between aortic root dimensions and moderate/ severe aortic regurgitation derived from multivariate logistic regression analysis. Odds ratios derived from multivariate logistic regression analysis adjusted for age, sex, body surface area, hypertension, and diabetes with Holm corrected p-values. Squares and horizontal

group, especially in those presenting with a moderate and severe AR. However, when controlling for age, BSA, and hypertension, this association did not remain statistically significant. Thus, the predominance of AR in male subjects might be primarily due to the higher prevalence of classical risk factors in males. Besides age and sex distribution, the improvement of echocardiographic technology might be causal for the relatively high AR prevalence in our study cohort since several of the mentioned studies are rather outdated. In particular, the high amount of mild AR cases might reflect a greater ability of current-era echocardiography equipment to detect small regurgitant jets. Furthermore, other comparable community based studies, as the OxVALVE Population Cohort Study (PCS), excluded subjects with known pre-existing valvular heart disease, resulting in a slightly lower rate of AR [19]. In summary, $\mathrm{AR}$ is a frequent finding at the populational-level, while its prevalence varies according to the analyzed study population and the utilized echocardiographic equipment. lines represent odds ratios and $95 \%$ confidence intervals. Ao aortic, $A R$ aortic regurgitation, $A s c$ ascending, $C I$ confidence interval, $E D$ end-diastolic, $M S$ mid-systolic, $O R$ odds ratio, $S T J$ sinotubular junction

\section{Aortic root size and AR}

In the present study, all mid-systolic and end-diastolic aortic root diameters were larger with increasing severity and prevalence of AR. A significant correlation between aortic root size and AR has been previously demonstrated by several research groups $[1-3,7,16,20]$. The AV is mounted in the complex structure of the aortic annulus that is tightly integrated into the functional unit of the aortic root. Therefore, dilatation of the entire aortic root or of its separate components may result in AV annulus dysfunction and subsequent development of AR. However, previous studies focused predominantly on the aortic root as one entity, without analyzing the separate components or the timepoint of measurement within the cardiac cycle. Therefore, we aimed to address this issue by examining all integral aortic root parts in systole and diastole. In adjusted binary logistic regression analysis, all individual parts of the aortic root were associated with 
moderate/severe AR. Mid-systolic measurements showed a tendency towards a stronger correlation with AR when compared to end-diastolic measurements. According to current recommendations by the ASE and EACVI, enddiastole is the proposed timepoint for all aortic measurements, except the AoAn [9]. Still, limiting the assessment solely to end-diastolic measurements does not comply with the complex interplay of the aortic root with pressure changes during the cardiac cycle which ensures AV integrity. In this regard, our data suggest a correlation of the same magnitude between AR and mid-systolic as compared to end-diastolic aortic root diameters. This finding can be explained by the fact that HCHS participants were at moderate to advanced age and had a "normal" tricuspid AV. These selection criteria resulted in an exclusion of most subjects with a dilated AoAn, who frequently present at a young age (i.e., congenital connective tissue disorders) and those with congenital AV lesions (i.e., bicuspid AV). Previous data demonstrated that the age-dependent increase in diameter is more prevalent in STJ as compared to AoAn [21]. Therefore, the likely explanation for the strongest correlation between AR severity and STJ diameter is given by the fact that our middle-aged /older study cohort with tricuspid AV undergoes a progressive STJ dilatation due to ageing-dependent remodeling, while those subjects with primarily dilated AoAn are predominantly excluded. The functional impact of STJ dilatation on the prevalence and the severity of AR results from the radial displacement of AV commissures creating a central coaptation defect with consecutive AR. The normalization of STJ diameter by surgical STJ annuloplasty or replacement of the ascending aortic aneurysm corrects the commissural displacement and is followed by improved AV competence.

Several previous studies focused on the definition of cut-off values of aortic root size which would be associated with AR occurrence [22]. Roman et al. and Seder et al. defined a threshold of $4.5 \mathrm{~cm}$ and $4.3 \mathrm{~cm}$ for the SoV diameter which were associated with a $100 \%$ prevalence of AR [1,2]. Both studies used M-Mode echocardiography limiting the translation of these findings into the HCHS study design. Nonetheless, we were able to confirm and augment previous findings by demonstrating a linear correlation between aortic root dimensions and AR prevalence as well as an association between aortic root size and AR severity. However, due to the functional impact of several AV components on AV function and the development of AR (i.e., aortic cusps, AoAn, STJ) a simplified use of aortic root size to predict AR severity is rather counterproductive. The focus should rather be on the separate components of AV complex that determine $\mathrm{AV}$ function and their interaction.

\section{Limitations}

The results of this study are influenced by the subjects included. Our study cohort represents the first 10.000 subjects of the HCHS. Most of the subjects were middle-aged and free of symptoms of cardiovascular disease. Therefore, the translation into other populations and utilization in the clinical setting is limited. Only a small proportion of the overall cohort showed severe AR. Although our study is derived from a large sample, most subjects showed mild AR, which led to an underrepresentation of the moderate/ severe group. The degree of association of the different aortic root components with AR severity could vary considerably according to patients' baseline characteristics. The presented regression analysis was adjusted for all detectable confounding variables. Nevertheless, we cannot exclude that other relevant interacting variables were not considered. The pathogenetic mechanism by which STJ diameter is associated with AR was not addressed. Whether AR itself induced the aorta to dilate or aortic root dilatation led to regurgitation was not answered. Most importantly, our study was not designed to investigate the causality of AR, which is not possible due to cross-sectional study design, but to evaluate the correlation between aortic root diameters and AR severity.

\section{Conclusion}

In a large population-based sample, AR prevalence was $14.2 \%$, with $2.8 \%$ being moderate/severe AR. Higher aortic root diameters at all levels were significantly associated with the prevalence and severity of AR. Of those, mid-systolic STJ had the strongest association with moderate/severe AR, reflecting its pathophysiological role in the context of an ageing aorta. Clinicians should closely monitor aortic root dilatation in the context of AR severity. Special focus should be paid to STJ dilatation as it showed the strongest association with a moderate/severe AR. Further validation in a broader, prospective population-based sample to evaluate the prognostic role of dilated aortic root diameters in the context of AR is needed.

Supplementary Information The online version contains supplementary material available at https://doi.org/10.1007/s10554-021-02337-6.

Acknowledgements The authors acknowledge the participants of the Hamburg City Health Study, the staff at the Epidemiological Study Center, cooperation partners, patrons and the Deanery from the University Medical Center Hamburg.

Funding Open Access funding enabled and organized by Projekt DEAL. The HCHS is supported by the Innovative medicine initiative 
[Grant Number 116074], by the Foundation Leducq [Grant Number 16 CVD 03], by the euCanSHare grant agreement [Grant Number 825903-euCanSHare H2020], and the Deutsche Forschungsgemeinschaft [Grant Number TH1106/5-1; AA93/2-1]. Furthermore, it is supported by the participating institutes and departments from the University Medical Centre Hamburg-Eppendorf, which contribute with individual and scaled budgets to the overall funding. Technical equipment is provided by SIEMENS according to a contract for 12 years, the Schiller AG on a loan basis for six years, and Topcon on a loan basis from 2017 until 2022. The Hamburg City Health Study is additionally supported by an unrestricted grant (2017 to 2022) by Bayer. Project-related analyses are supported by Amgen, Astra Zeneca, BASF, Deutsche Gesetzliche Unfallversicherung (DGUV), Deutsches Krebsforschungszentrum (DKFZ), Deutsches Zentrum für Herz-KreislaufForschung (DZHK), Deutsche Stiftung für Herzforschung, Novartis, Seefried Stiftung, and Unilever. The study is further supported by donations from the "Förderverein zur Förderung der HCHS e.V.", TePe ${ }^{\circledR}$ (2014) and Boston Scientific (2016). A current list of the supporters is online available on www.uke.de/hchs. Sponsor funding has in no way influenced the content or management of this study.

Data availability The data underlying this article cannot be shared publicly due to the privacy of individuals that participated in the study. The data will be shared on reasonable request to the corresponding author.

Code availability Will be shared on reasonable request. List of used $\mathrm{R}$ packages: forestplot (1.10); ggpubr (0.2.4); gridExtra (2.3); reshape2 (1.4.3); survival (3.1-8); tableone (0.10.0); tidyverse (1.2.1)

\section{Declarations}

Conflict of interest EG reports honoraria from Edwards, Medtronic, LivaNova, and Aesculap, outside of the submitted work. SB reports honoraria from Abbott, Siemens, Thermo Fisher, and Roche, outside of the submitted work.

Ethical approval The authors do hereby declare that their study complies with the Declaration of Helsinki.

Illustrations and figures The authors do hereby declare that all illustrations and figures in the manuscript are entirely original and do not require reprint permission.

Open Access This article is licensed under a Creative Commons Attribution 4.0 International License, which permits use, sharing, adaptation, distribution and reproduction in any medium or format, as long as you give appropriate credit to the original author(s) and the source, provide a link to the Creative Commons licence, and indicate if changes were made. The images or other third party material in this article are included in the article's Creative Commons licence, unless indicated otherwise in a credit line to the material. If material is not included in the article's Creative Commons licence and your intended use is not permitted by statutory regulation or exceeds the permitted use, you will need to obtain permission directly from the copyright holder. To view a copy of this licence, visit http://creativecommons.org/licenses/by/4.0/.

\section{References}

1. Roman MJ, Devereux RB, Niles NW, Hochreiter C, Kligfield P, Sato N, Spitzer MC, Borer JS (1987) Aortic root dilatation as a cause of isolated, severe aortic regurgitation Prevalence, clinical and echocardiographic patterns, and relation to left ventricular hypertrophy and function. Ann Intern Med 106(6):800-807. https://doi.org/10.7326/0003-4819-106-6-800

2. Seder JD, Burke JF, Pauletto FJ (1990) Prevalence of aortic regurgitation by color flow Doppler in relation to aortic root size. J Am Soc Echocardiogr 3(4):316-319. https://doi.org/10.1016/s08947317(14)80315-5

3. Lebowitz NE, Bella JN, Roman MJ, Liu JE, Fishman DP, Paranicas M, Lee ET, Fabsitz RR, Welty TK, Howard BV, Devereux RB (2000) Prevalence and correlates of aortic regurgitation in American Indians : the strong heart study. J Am Coll Cardiol 36(2):461-467. https://doi.org/10.1016/S0735-1097(00)00744-0

4. Babaee Bigi MA, Aslani A (2007) Aortic root size and prevalence of aortic regurgitation in elite strength trained athletes. Am J Cardiol 100(3):528-530. https://doi.org/10.1016/j.amjcard.2007.02. 108

5. Guiney TE, Davies MJ, Parker DJ, Leech GJ, Leatham A (1987) The aetiology and course of isolated severe aortic regurgitation: a clinical, pathological, and echocardiographic study. Heart 58(4):358-368. https://doi.org/10.1136/hrt.58.4.358

6. Roman MJ, Devereux RB, Kramer-Fox R, O'Loughlin J (1989) Two-dimensional echocardiographic aortic root dimensions in normal children and adults. Am J Cardiol 64(8):507-512

7. Hahn RT, Roman MJ, Mogtader AH, Devereux RB (1992) Association of aortic dilation with regurgitant, stenotic and functionally normal bicuspid aortic valves. J Am Coll Cardiol 19(2):283-288. https://doi.org/10.1016/0735-1097(92)90479-7

8. Jagodzinski A, Johansen C, Koch-Gromus U, Aarabi G, Adam G, Anders S, Augustin M, Kellen RB, Beikler T, Behrendt CA, Betz CS, Bokemeyer C, Borof K, Briken P, Busch CJ, Büchel C, Brassen S, Debus ES, Eggers L, Fiehler J, Gallinat J, Gellißen S, Gerloff C, Girdauskas E, Gosau M, Graefen M, Härter M, Harth V, Heidemann C, Heydecke G, Huber TB, Hussein Y, Kampf MO, Knesebeck O, Konnopka A, König HH, Kromer R, Kubisch C, Kühn S, Loges S, Löwe B, Lund G, Meyer C, Nagel L, Nienhaus A, Pantel K, Petersen E, Püschel K, Reichenspurner H, Sauter G, Scherer M, Scherschel K, Schiffner U, Schnabel RB, Schulz H, Smeets R, Sokalskis V, Spitzer MS, Terschüren C, Thederan I, Thoma T, Thomalla G, Waschki B, Wegscheider K, Wenzel JP, Wiese S, Zyriax BC, Zeller T, Blankenberg S (2020) Rationale and design of the Hamburg City Health Study. Eur J Epidemiol 35(2):169-181. https://doi.org/10.1007/s10654-019-00577-4

9. Lang RM, Badano LP, Mor-Avi V, Afilalo J, Armstrong A, Ernande L, Flachskampf FA, Foster E, Goldstein SA, Kuznetsova T, Lancellotti P, Muraru D, Picard MH, Rietzschel ER, Rudski L, Spencer KT, Tsang W, Voigt JU (2015) Recommendations for cardiac chamber quantification by echocardiography in adults: an update from the American society of echocardiography and the European association of cardiovascular imaging. J Am Soc Echocardiogr 16(3):233-271. https://doi.org/10.1093/ehjci/jev014

10. Goldstein SA, Evangelista A, Abbara S, Arai A, Asch FM, Badano LP, Bolen MA, Connolly HM, Cuéllar-Calàbria H, Czerny M, Devereux RB, Erbel RA, Fattori R, Isselbacher EM, Lindsay JM, McCulloch M, Michelena HI, Nienaber CA, Oh JK, Pepi M, Taylor AJ, Weinsaft JW, Zamorano JL, Dietz H, Eagle K, Elefteriades J, Jondeau G, Rousseau H, Schepens M (2015) Multimodality imaging of diseases of the thoracic aorta in adults: from the American Society of Echocardiography and the European Association of Cardiovascular Imaging. J Am Soc Echocardiogr 28(2):119-182. https://doi.org/10.1016/j.echo.2014.11.015

11. Eacts C, Thoracic S, Germany CH, Rosenhek R, France EL, Rodriguez D, Tornos P, Vahanian A, Luis J, Delgado V, Uk D.F. \& France T.F (2017) 2017 ESC / EACTS Guidelines for the management of valvular heart disease The Task Force for the Management of Valvular Heart Disease of the European Society of 
Cardiology (ESC ) and the European. Eur Heart J 38:2739-2786. https://doi.org/10.1093/eurheartj/ehx391

12. Berger M, Hecht SR, Tosh A, Lingam U (1989) Pulsed and continuous wave Doppler echocardiographic assessment of valvular regurgitation in normal subjects. J Am Coll Cardiol 13(7):1540 1545. https://doi.org/10.1016/0735-1097(89)90345-8

13. Klein AL, Burstow DJ, Tajik AJ, Zachariah PK, Taliercio CP, Taylor CL, Bailey KR, Seward JB (1990) Age-related prevalence of valvular regurgitation in normal subjects: a comprehensive color flow examination of 118 volunteers. J Am Soc Echocardiogr 3(1):54-63. https://doi.org/10.1016/s0894-7317(14)80299-x

14. Jobic Y, Slama M, Tribouilloy C, Lan Cheong Wah L, Choquet D, Boschat J, Penther P, Lesbre JP (1993) Doppler echocardiographic evaluation of valve regurgitation in healthy volunteers. Br Heart J 69(2):109-113. https://doi.org/10.1136/hrt.69.2.109

15. Singh JP, Evans JC, Levy D, Larson MG, Freed LA, Fuller DL, Lehman B, Benjamin EJ (1999) Prevalence and clinical determinants of mitral, tricuspid, and aortic regurgitation (the Framingham Heart Study). Am J Cardiol 83(6):897-902. https://doi.org/ 10.1016/s0002-9149(98)01064-9

16. Reid CL, Anton-Culver H, Yunis C, Gardin JM (2007) Prevalence and clinical correlates of isolated mitral, isolated aortic regurgitation, and both in adults aged 21 to 35 years (from the CARDIA study). Am J Cardiol 99(6):830-834. https://doi.org/10.1016/j. amjcard.2006.10.048

17. Stefano G, Fox K, Schluchter M, Hoit BD (2008) Prevalence of unsuspected and significant mitral and aortic regurgitation. J Am Soc Echocardiogr 21(1):38-42. https://doi.org/10.1016/j.echo. 2007.05.006

18. de Heer F, Kluin J, Elkhoury G, Jondeau G, Enriquez-Sarano M, Schäfers HJ, Takkenberg JJM, Lansac E (2019) AVIATOR: an open international registry to evaluate medical and surgical outcomes of aortic valve insufficiency and ascending aorta aneurysm.
J Thorac Cardiovasc Surg 157(6):2202-2211.e7. https://doi.org/ 10.1016/j.jtcvs.2018.10.076

19. d'Arcy JL, Coffey S, Loudon MA, Kennedy A, Pearson-Stuttard J, Birks J, Frangou E, Farmer AJ, Mant D, Wilson J, Myerson SG, Prendergast BD (2016) Large-scale community echocardiographic screening reveals a major burden of undiagnosed valvular heart disease in older people: the OxVALVE Population Cohort Study. Eur Heart J 37(47):3515-3522. https://doi.org/10.1093/eurheartj/ ehw229

20. Palmieri V, Bella JN, Arnett DK, Roman MJ, Oberman A, Kitzman DW, Hopkins PN, Paranicas M, Rao DC, Devereux RB (2012) Aortic root dilatation at sinuses of valsalva and aortic regurgitation in hypertensive and normotensive subjects. Hypertension 37(5):1229-1235. https://doi.org/10.1161/01.hyp.37.5. 1229

21. Saura D, Dulgheru R, Caballero L, Bernard A, Kou S, Gonjilashvili N, Athanassopoulos GD, Barone D, Baroni M, Cardim N, Hagendorff A, Hristova K, Lopez T, La Morena G, De PBA, Penicka M, Ozyigit T, Carbonero JDR, Van De VN, Von BRS, Vinereanu D, Zamorano JL, Gori AS, Cosyns B, Donal E, Habib G, Addetia K, Lang RM, Badano LP, Lancellotti P (2017) Twodimensional transthoracic echocardiographic normal reference ranges for proximal aorta dimensions: results from the EACVI NORRE study. Eur Heart J Cardiovasc Imaging 18(2):167-179. https://doi.org/10.1093/ehjci/jew053

22. Atsuchi Y, Nagai Y, Komatsu Y, Nakamura K, Shibuya M, Hirosawa K (1977) Echocardiographic manifestation of annuloaortic ectasia: its "paradoxical" motion of the aorta and premature systolic closure of the aortic vale. Am Heart J 93(4):428-433. https:// doi.org/10.1016/s0002-8703(77)80404-3

Publisher's Note Springer Nature remains neutral with regard to jurisdictional claims in published maps and institutional affiliations. 